\title{
Assessment of Underwater Glider Performance Through Viscous Computational Fluid Dynamics
}

\author{
Artur K. Lidtke, Stephen R. Turnock and Jon Downes \\ Fluid-Structure Interactions Group, University of Southampton \\ Southampton, SO16 7QF, United Kingdom \\ E-mail: ak11g09@soton.ac.uk
}

\begin{abstract}
The process of designing an apt hydrodynamic shape for a new underwater glider is discussed. Intermediate stages include selecting a suitable axi-symmetric hull shape, adding hydrofoils and appendages, and evaluating the performance of the final design. All of the hydrodynamic characteristics are obtained using computational fluid dynamics using the $k_{T}-k_{L}-\omega$ transition model. It is shown that drag reduction of the main glider hull is of crucial importance to the ultimate performance. Suggested steps for achieving this are the encouragement of natural laminar flow, integration of sensors into the streamlined hull shape, and sound operational practice.
\end{abstract}

\section{INTRODUCTION}

Underwater gliders are autonomous underwater vehicles (AUV) which change their potential energy by adjusting their displacement via the use of a buoyancy engine and utilise the resulting vertical motion to generate lift, a fraction of which delivers a forward thrust force. This principle of operation is illustrated in Figure 1. The mode of propulsion employed by this category of AUVs only requires direct actuation at the top and bottom of each tack when extra buoyancy is being added or subtracted from the vehicle [1], [2] which implies low power consumption. Consequently, underwater glider have seen wide use in high-endurance applications with deployments lasting of the order of months and individual vehicles travelling distances as long as several thousand miles [1]. Given the duration of their missions and nature of their service underwater gliders are limited in terms of what sensors they may feasibly carry. Nonetheless, operators and designers, incentivised by potential savings in research ship time, are constantly looking at deploying innovative payloads increasing the scope of application of gliders to various aspects of oceanographic research.

Forward velocities of most gliders typically do not exceed $0.5 \mathrm{~ms}^{-1}$ due to the focus on promoting energy efficiency through the use of modest-sized buoyancy engines rather than at maximising their speed [3], [4], [5], [1]. This translates to Reynolds numbers of the order of 1 million for a 2 metre hull and less than 100,000 for hydrofoils with high aspect ratios which are typically seen on most designs [1], [6]. Therefore natural laminar flow may play an important role in determining the performance of these key glider components.

A critical consideration for an underwater glider is the glide path angle it adopts during its ascent or descent. A well understood trade-off in operating these vehicles is that minimising the glide path angles leads to increased range but also reduces the component of net buoyancy or gravity force acting against the vehicle drag and hence may lead to a reduced speed [7], [4]. The former is primarily due to the fact that between 60 and $80 \%$ of the energy usage on an underwater glider is attributed to operation of the buoyancy engine [4], [1], [5] and so fewer dives mean longer battery life. Shallow glide path angles are also generally associated with higher angles of attack [7] which may lead to laminar separation on the hydrofoil sections and the hull itself [8] and thus causing Reynolds number-dependence of the vehicle performance. Another practical reason for maintaining high glide path angles is that it is desirable to sample the data from most of the scientific sensors along the natural vertical gradients of the environmental quantities being measured [5]. For these reasons most oceanographic gliders, while capable of travelling at glide path angles as low as 10 to $15^{\circ}[7],[1]$, typically operate at slopes around 35 to $40^{\circ}$ [4]. A notable exemption is the XRay blended wing glider which was designed for high transit speeds and long range without consideration for vertical data profiling and hence operates at glide path slopes as high as 10 to 1 [2].

The relatively high glide path angles required of oceanographic gliders also bears implications for the hydrodynamic efficiency required of them to fulfil their mission as the glider's lift-to-drag (L/D) ratio is directly linked to the glide paths it may adopt [4]. For instance, modern gliders used in air, or sail planes, are generally required to travel large horizontal distances per unit height they lose due to gravity and, as a

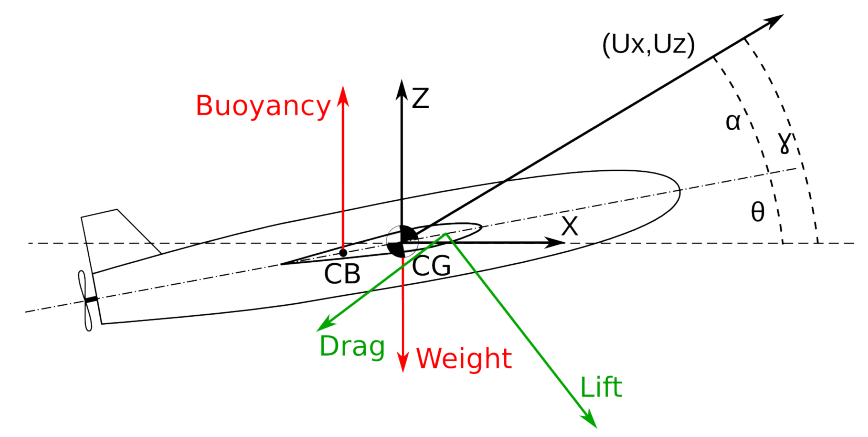

Fig. 1. Schematic representation of the principle of operation of an underwater glider. $\theta, \alpha$, and $\gamma$ are the pitch, angle of attack, and glide path angles, respectively (forces and moments exaggerated for clearer presentation). 
consequence, are designed to have high overall L/D in excess of 20 [4]. Most underwater gliders, on the other hand, develop $\mathrm{L} / \mathrm{D}$ in the range of 1.5 to 5 [4], [1] which is sufficient to meet their mission requirements. The design implication is that only a relatively small wing area is needed per unit of hull drag. Bearing in mind that a large proportion of the overall lift the vehicle produces acts against the net buoyancy force, it follows that minimising the overall system drag, and therefore the wing area within the constraints imposed by operational requirements, is a key target for extending the range of the glider by reducing the necessary size of the buoyancy engine.

Reduction of drag may be achieved, for instance, by counteracting marine growth of the body of the glider which has been reported to be a serious issue, particularly for long deployments in coastal waters [7], [1]. Another approach is to design small, compact glider shapes with small overall wetted surface area and to reduce the impact of sensors, protrusions and discontinuities on the overall resistance. The latter has been reported to be a crucial factor, often contributing to between 25 and $50 \%$ of the total drag [1], [9], [4], [10].

On the strictly hydrodynamic side, one may attempt to utilise the low Reynolds numbers seen of the gliders to encourage natural laminar flow (NLF), similarly to the solution adopted in the Seaglider [11], [9]. This solution poses several difficulties, for instance, designing a pressure vessel that fits into the unconventional shape and achieving surface finish and manufacturing tolerances accurate enough not to trip the flow into the turbulent regime too early. From the perspective of a designer proposing a sound hydrodynamic shape for a new underwater glider, a better understanding of the extent of the natural laminar flow is therefore of paramount importance if an efficient vehicle is to be developed.

The aim of this paper is to describe part of the preliminary design process of the hydrodynamic shape for a new underwater glider for oceanographic research. The present work contributes to the EU-funded BRIDGES consortium (http://www.bridges-h2020.eu/). Performance of the considered shapes is evaluated using computational fluid dynamic (CFD) simulations. While the concept of applying modern numerical techniques to study the flow past autonomous underwater vehicles is not new [10], [12], [13], [14], [15], [16], [17], most of the recent work has been focused on AUVs operating at Reynolds numbers higher than those typically seen by underwater gliders. An interesting observation is also that while older work utilised methods capable of accounting for transition to turbulence at least to a certain extent [11], [18], [8], the majority of the more recent papers relied on solving Reynolds Averaged Navier-Stokes equations using models recognised for their inability to tackle this complex physical phenomenon [19] with only a handful of exceptions [20]. In the present work the $k_{L}-k_{T}-\omega$ model by Walters and Cokkjat [21] is utilised in order to overcome the latter difficulty and provide more realistic performance estimates for the new underwater glider design.

First, the proposed numerical set-up is applied to canonical test cases of flow past a flat plate with and without a stream- wise pressure gradient (Ercoftac T3) [22], flow past a foil with laminar separation (SD7003) [23], and a low Reynolds number axi-symmetric shape (Hansen-Hoyt body) [24]. Then, four axi-symmetric hull shapes are considered over a range of conditions typical for an underwater glider in service. Tradeoffs each of them offer are discussed and most important flow features are presented. The most promising concept is then used to develop a range of practical hull shapes fitted with hydrofoils and other appendages. Their performance is then also evaluated and compared. Consequently, a design for a new underwater glider is proposed and its lift and drag characteristics are evaluated and discussed. These are translated into a performance envelope indicating possible speeds the design could achieve subject to a fixed buoyancy engine actuation.

\section{Methodology}

\section{A. Turbulence modelling}

All of the performed simulations are carried out using steady, incompressible Reynolds averaged Navier-Stokes (RANS),

$$
\begin{aligned}
\nabla \cdot \overline{\mathbf{U}} & =0, \\
(\overline{\mathbf{U}} \cdot \nabla) \overline{\mathbf{U}}+\nabla \cdot\left(\overline{\mathbf{u}^{\prime} \mathbf{u}^{\prime}}\right) & =-\frac{1}{\rho} \nabla \bar{p}+\nu \nabla^{2} \overline{\mathbf{U}}
\end{aligned}
$$

In the above $\mathbf{U}$ is the fluid velocity, $p$ is the pressure, $\rho$ is the density, and $\nu$ is the kinematic viscosity. The overline notation indicates an ensemble average and $\rho \nabla \cdot\left(\overline{\mathbf{u}^{\prime} \mathbf{u}^{\prime}}\right)$ is termed the Reynolds' stress tensor and represents the action of turbulence on the mean flow. Due to its non-linearity the latter term is modelled rather than solved for in order reduce the computational effort. The governing equations are solved using an implicit SIMPLE algorithm implemented in the OpenFOAM framework, a set of open-source libraries and utilities aimed at numerical solution of partial differential equations. The approach relies on discretising the equations using the finite volume method and solves them in an iterative manner.

To provide benchmark results, the Reynolds stress tensor is first modelled using the Menter $k-\omega S S T$ model [25], arguably the most common choice in simulating flow past underwater vehicles [16], [15], [14]. It uses two additional variables, the turbulent kinetic energy, $k$, and the specific dissipation rate, $\omega$, to provide expressions for the turbulent length and time scales. These are then used to yield a scalar variable, the turbulent viscosity $\nu_{T}$. This quantity aims to relate the rate of strain in the mean flow to the action of turbulence turbulence to the mean flow following the Boussinesq hypothesis. Exact details of the model are discussed by Menter [25]. This model does not account for the occurrence of transition well, effectively predicting fully turbulent flow over most of the tested geometries. From the perspective of underwater glider design, the results obtained using this approach may be viewed as a scenario in which local discontinuities, such as scratches, dirt, joints, etc., cause the flow to transition almost immediately. 
In order to predict transition to turbulence the $k_{T}-k_{L}-\omega$ model by Walters and Cokkjat [21] is also used in the present work. This model solves three transport equations for turbulent kinetic energy, $k_{T}$, specific dissipation rate, $\omega$, and laminar kinetic energy, $k_{L}$. The latter is used to determine where laminar instabilities render the flow turbulent and hence onset of transition may be captured. Fundamentally, this builds on similar principles to the Menter model although an additional transport equation for the laminar kinetic energy is solved, yielding

$$
\begin{aligned}
\frac{\mathrm{D} k_{T}}{\mathrm{D} t}= & P_{k_{T}}+R_{B P}+R_{N A T}-\omega k_{T}-D_{T} \\
& +\frac{\partial}{\partial x_{j}}\left[\left(\nu+\frac{\alpha_{T}}{\sigma_{K}}\right) \frac{\partial k_{T}}{\partial x_{j}}\right] \\
\frac{\mathrm{D} k_{L}}{\mathrm{D} t}= & P_{k_{L}}-R_{B P}-R_{N A T}-D_{L}+\frac{\partial}{\partial x_{j}}\left[\nu \frac{\partial k_{L}}{\partial x_{j}}\right], \\
\frac{\mathrm{D} \omega}{\mathrm{D} t}= & C_{\omega 1} \frac{\omega}{k_{T}} P_{k_{T}}-C_{\omega 2} \omega^{2}+C_{\omega 3} f_{\omega} \alpha_{T} f_{w}^{2} \frac{\sqrt{k_{T}}}{d^{3}} \\
& +\left(\frac{C_{\omega R}}{f_{W}}-1\right) \frac{\omega}{k_{T}}\left(R_{B P}+R_{N A T}\right) \\
& +\frac{\partial}{\partial x_{j}}\left[\left(\nu+\frac{\alpha_{T}}{\sigma_{w}}\right) \frac{\partial \omega}{\partial x_{j}}\right]
\end{aligned}
$$

where the terms on the right-hand-sides of the equations denote production, destruction and transport of the basic turbulent quantities. The source citation describes these in detail [21].

The key concepts of the present transition model are that the total kinetic energy of fluctuations in the flow, $k_{T}+k_{L}$, is assumed to be produced proportionally to the mean strain rate in the fluid. Linear coefficient, $P_{k_{L}}=\nu_{T, 1} S^{2}$, is also assumed to govern this relationship.

\section{B. Mesh generation}

Unappended glider hull simulations are performed using structured hexahedral grids. Due to more complex geometry, the proposed glider shape including foils, tail fin, and stabilisers, has been meshed using unstructured tetrahedra meshes with prism boundary layer cells. An example of such a grid is presented in Figure 2. The outer domain shape (seen in Figure 2a) is a hemisphere with radius equal to 10 vehicle lengths. Approximately 3 million cells are used to resolve the flow around half of the vehicle in the scenarios where fully-appended designs are being considered. All meshes are generated using Pointwise [26] and are design to meet the $y^{+} \leq 1$ criterion needed to resolve the inner regions of the boundary layers.

\section{Simulation set up}

In the governing flow equations, the convective terms are discretised using second-order upwind scheme and turbulent quantities are treated with first-order upwind stencils. Steady flow is assumed and hence the time derivatives are dropped. Coupling between the pressure and velocity is solved using an implicit, segregated SIMPLE algorithm. The systems of

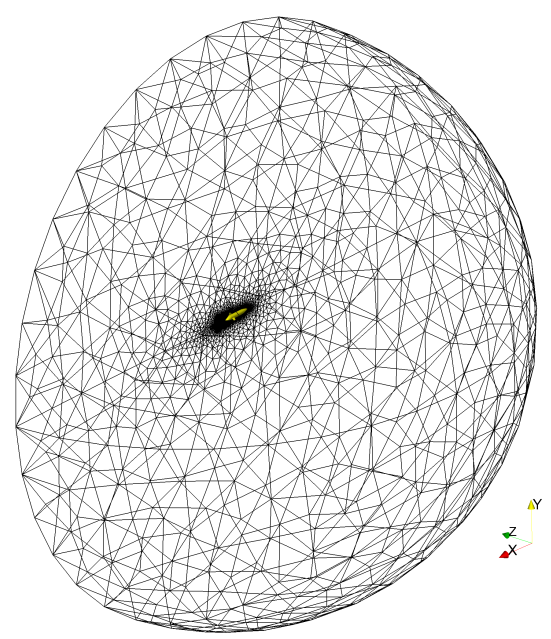

(a) Domain

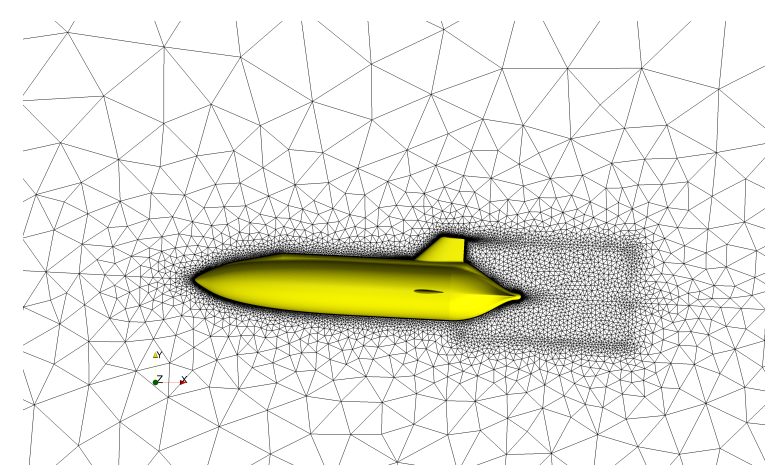

(b) Free-stream near the hull

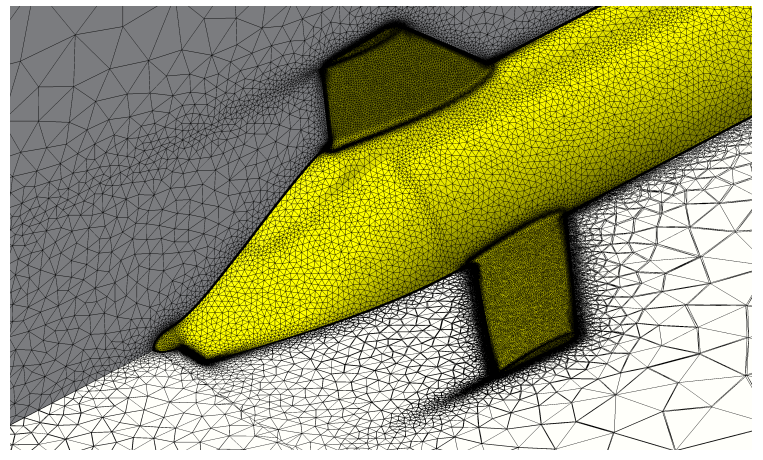

(c) Stern area

Fig. 2. View of the unstructured, tetrahedral mesh used to compute the flow around the underwater glider.

equations are solved until convergence of $\mathrm{L}_{1}$ norm to at least $10^{-6}$ has been achieved. The fully-appended hull simulations required approximately 48 hours to evaluate a single operational point on 32 processors.

In all of the simulations inlet turbulence levels of $0.2 \%$ are used with eddy viscosity ratios of approximately 100 . These correspond to typical high-quality wind tunnel conditions, although the values encountered by the glider in service may vary depending on the environment it operates in and future sensitivity studies will need to be carried out to better under- 
stand the effect of these quantities on achieved performance. For each case a symmetry plane condition is used to allow only half of the design to be simulated, thus reducing the cell count substantially. A no-slip boundary condition is applied on the glider and all its appendages while free-stream inlet-outlet conditions is applied to the hemispherical outer domain.

\section{Validation}

An extensive validation study has been performed in order to provide confidence and establish the range of validity of the predictions made. For brevity only key aspects of results are presented here. Each of the discussed test cases is considered to be well-established and the respective source citations provide additional information about the experimental studies.

Figure 3 presents the skin friction coefficient distribution over a flat plate with and without stream-wise pressure gradient, experimental data for which was reported by Coupland $e t$ al. [22]. The case covered a wide range of inlet turbulence intensities and Reynolds numbers, making it appealing to the current application in which both low (appendages) and intermediate (hull) Reynolds number regimes are expected. The worst agreement with the experiment may be seen for the $\mathrm{T} 3 \mathrm{C} 1$ and $\mathrm{T} 3 \mathrm{C} 4$ cases, which are characterised by very high inlet turbulence intensity $(9 \%)$ and very low Reynolds number $(13,000)$, respectively. The more moderate cases, more representative of the conditions expected in reality, show satisfactory agreement.

Figure 4 presents pressure coefficient distribution computed for an axi-symmetric body of revolution intended to encourage natural laminar flow, as discussed by Dodbele and van Dam [24]. The computational results show similar trends to the ones reported in the experiments, although the high pressure peak at $x / L$ of 0.6 and the low pressure trough at $x / L ~ 0.8$ are slightly under-predicted.

Figure 5 depicts the stream-wise velocity contours predicted for the SD7003 foil with experimental data by $\mathrm{Ol}$ et al. [23]. It may be seen that the onset of laminar separation as well as transition occurring on top of the separation bubble are predicted quite accurately. The extent of the separation bubble predicted by the present model is greater than was reported in the experiments, however.

Finally, Figure 6 shows the predicted lift coefficients for a symmetric low-Re airfoil section, SD8020 [27]. Xfoil predictions for this and another symmetric section, the J5012, are also shown. It may be seen that the trend of the lift coefficient caused by laminar separation is represented well by the present model, although the sudden jump in lift at an angle of attack of $1^{\circ}$ is instead captured as a gradual increase and the final level of the lift curve is higher than was reported in the experiments. The present model provides a slight improvement over the Xfoil predictions, although it is based on completely different assumptions and may be applied even to complex, 3D geometries.

The presented validation study has indicated that the current numerical set up models the principle physics involved in natural transition well across a range of flow regimes representative

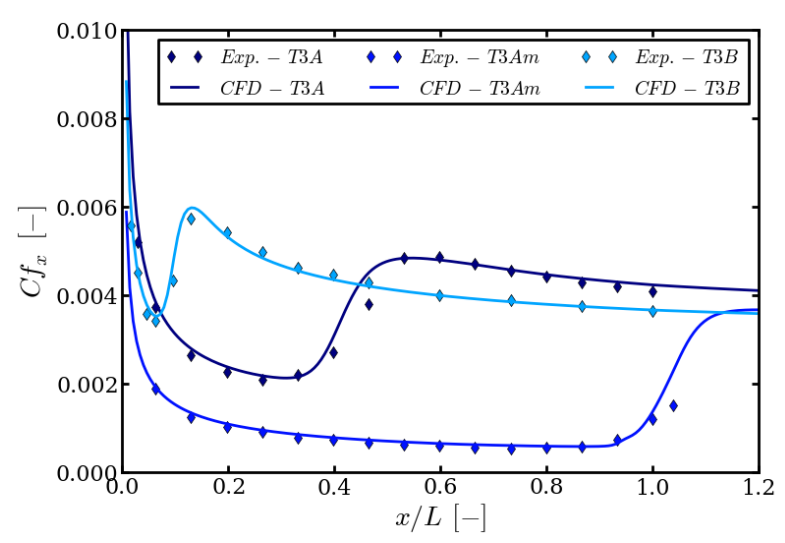

(a) Zero pressure gradient (ZPG)

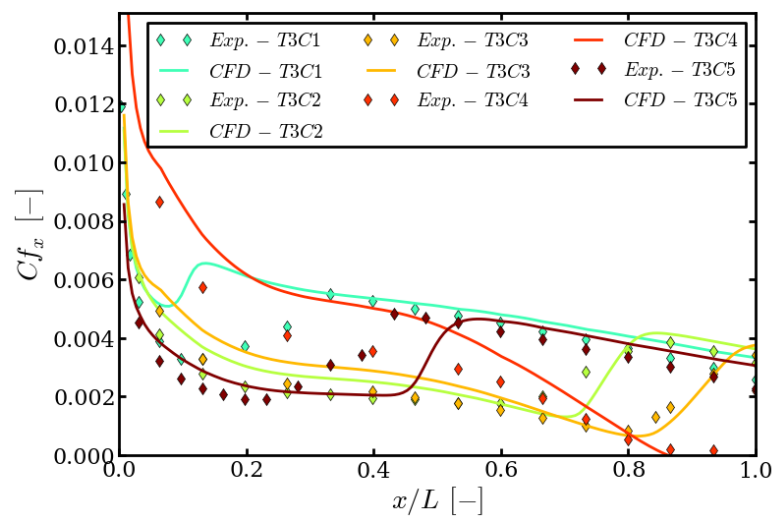

(b) Variable stream-wise pressure gradient

Fig. 3. Skin friction coefficient distribution for the T3 test case [22]. Reynolds numbers between 13,000 and 2 million.

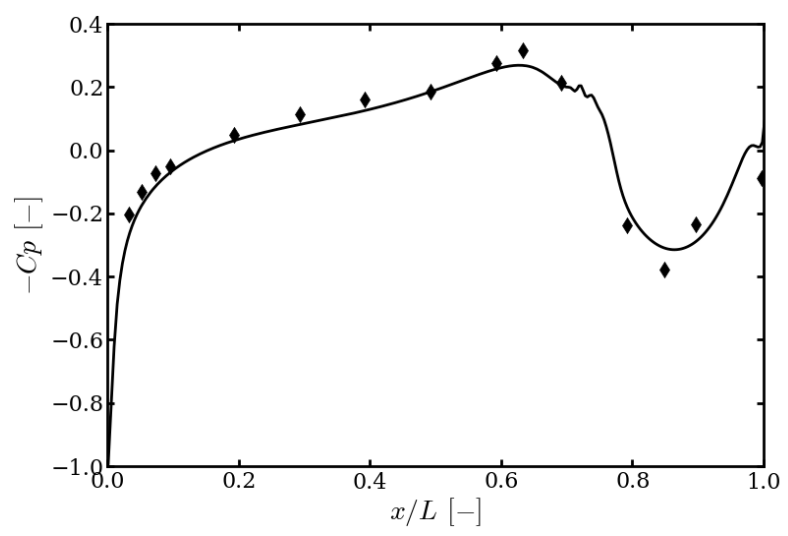

Fig. 4. Stream-wise pressure coefficient distribution on an axi-symmetric natural laminar flow (NLF) body computed using the present transition model [24]. Reynolds number 1.2 million. 


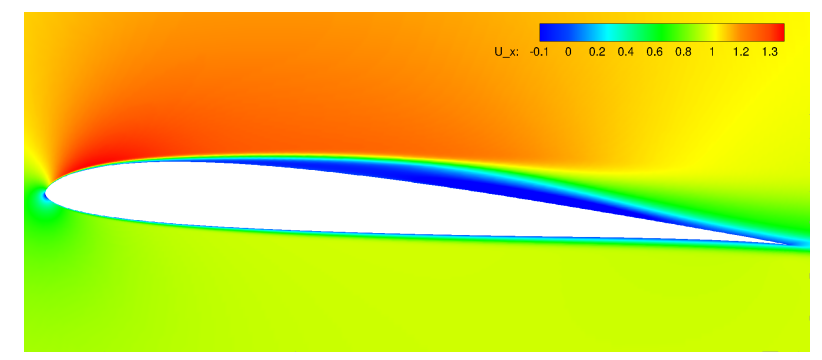

(a) $\mathrm{CFD}$

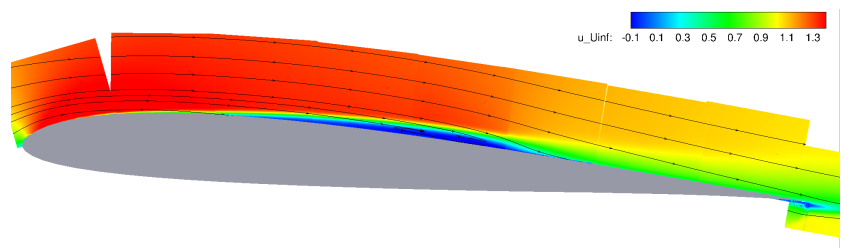

(b) Experiment (gaps correspond to lack of PIV data coverage)

Fig. 5. Stream-wise velocity iso-contours showing laminar separation on the SD7003 foil [23]. Reynolds number 40,000.

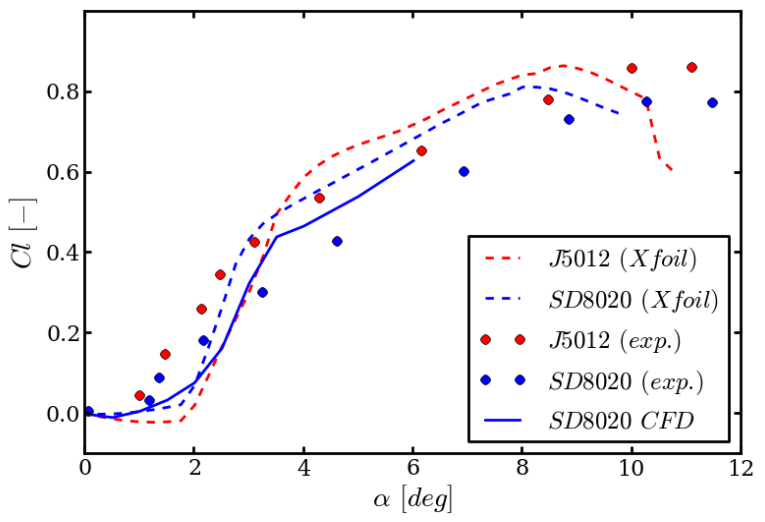

Fig. 6. Lift coefficient curves predicted using the current transition model compared against Xfoil and experimental data by Selig et al. [27]. All foils at $\operatorname{Re} 40,000$.

of what underwater gliders may experience in service. Most importantly, it has been able to predict the force coefficients acting on streamlined shapes and accounts for the effect of stream-wise pressure gradient. These two features are of key importance to being able to use the model to select more hydrodynamically sound designs.

\section{RESUlTS AND Discussion}

\section{A. Hull form shape selection}

Figure 7 presents candidate designs used to evaluate the effect of the underlying axi-symmetric part of the glider hull on the overall system performance. Variant 0 is a Myring body [28] with parallel sides and a conical stern, similar to several commercially available AUVs and underwater gliders. Designs 1 and 2 provide identical enclosed volume but have the maximum cross-section area point shifted aft in order to induce a favourable pressure gradient over a large proportion of the hull, thus encouraging natural laminar flow [24]. Design

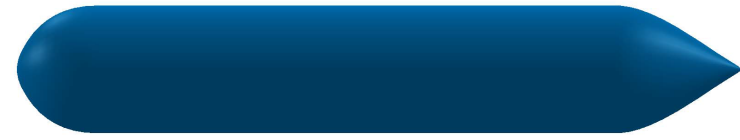

(a) Design 0

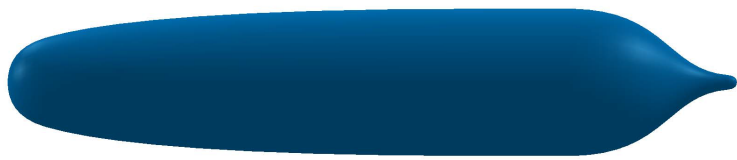

(b) Design 1

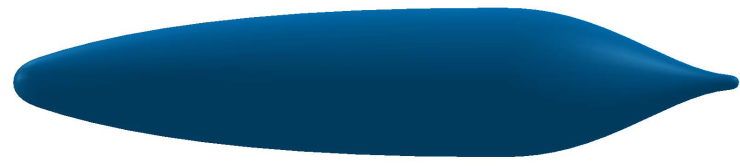

(c) Design 2

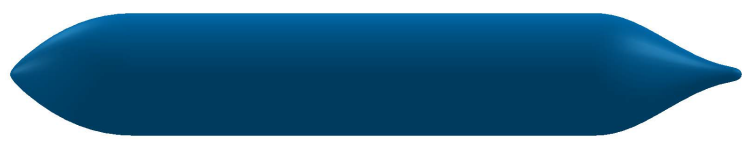

(d) Design 3

Fig. 7. Concepts of initial axi-symmetric hull designs (flow from left to right).

3 is a more streamlined Myring shape. Each of the hulls was designed to house a pressure vessel of given size, to meet length and width constraints and to provide enough useful volume for sensors and other pressure-resistant subsystems.

Flow past each of the designs was computed over a range of Reynolds numbers corresponding to nominal and maximum speeds and an angle of attack of 3 degrees. The latter was chosen as the target value for the glider in order to maximise performance of the hydrofoils based on 2D section characteristics.

Table I presents the lift and drag coefficients of the considered designs. It can be seen that design 2 has been predicted to produce both more lift and have a lower drag than the other design variants, despite a relatively high surface area. This is because of the favourable pressure gradient encouraging laminar flow all the way up to the maximum cross-section area, thus limiting the friction drag which constitutes approximately $70 \%$ of the total resistance. At the nominal Reynolds number, other designs offer comparable performance in terms of liftto-drag ratio. At the higher speed, design 1 suffers from significant flow separation on the suction surface leading to a negative lift coefficient. In this condition design 3 experiences slightly less drag than design 0 but produces up to five times less lift.

Figure 8 presents an example result of the stream-wise velocity contours predicted using the transition model for designs 0 and 2. It is worth noting how the natural laminar flow design only has one inflection point, which leads to a steady, favourable pressure gradient over most of the hull while the Myring shape induces low pressure regions at both ends of the parallel mid-body. This causes the flow to transition much sooner than for the former design, leading to a thick, turbulent 
TABLE I

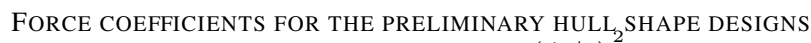
ASSUMING FREE TRANSITION (DEFINED USING $(\Delta / \rho)^{\frac{2}{3}}$ ) AT AN ANGLE OF ATTACK OF $3^{\circ}$

\begin{tabular}{|c|c|c|c|c|}
\hline Design & $\mathrm{C}_{\mathrm{D}}$ & $\mathrm{C}_{\mathrm{Di}}$ & $\mathrm{C}_{\mathrm{L}}$ & $\mathrm{L} / \mathrm{D}$ \\
\hline \multicolumn{5}{|c|}{$\operatorname{Re} 1.5$ million } \\
\hline 0 & $4.49 \mathrm{E}-3$ & 9.37E-5 & $4.40 \mathrm{E}-4$ & 0.10 \\
\hline 1 & $4.83 \mathrm{E}-3$ & $1.86 \mathrm{E}-4$ & $6.81 \mathrm{E}-4$ & 0.14 \\
\hline 2 & $2.14 \mathrm{E}-3$ & $1.20 \mathrm{E}-4$ & $1.26 \mathrm{E}-3$ & 0.59 \\
\hline 3 & $3.14 \mathrm{E}-3$ & $4.46 \mathrm{E}-3$ & $7.62 \mathrm{E}-4$ & 0.10 \\
\hline \multicolumn{5}{|c|}{ Re 3.0 million } \\
\hline 0 & $4.22 \mathrm{E}-3$ & $3.97 \mathrm{E}-5$ & $8.43 \mathrm{E}-4$ & 0.20 \\
\hline 1 & $2.23 \mathrm{E}-3$ & $-1.28 \mathrm{E}-3$ & 4.30E-4 & -0.48 \\
\hline 2 & $1.51 \mathrm{E}-3$ & $1.36 \mathrm{E}-4$ & $7.49 \mathrm{E}-4$ & 0.50 \\
\hline 3 & $4.02 \mathrm{E}-3$ & $2.94 \mathrm{E}-3$ & $2.83 \mathrm{E}-4$ & 0.04 \\
\hline
\end{tabular}

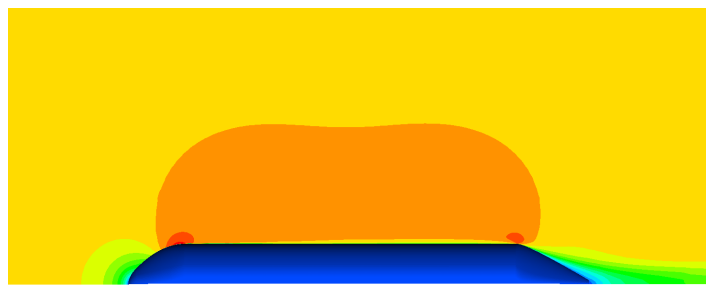

(a) Design 0 - Myring

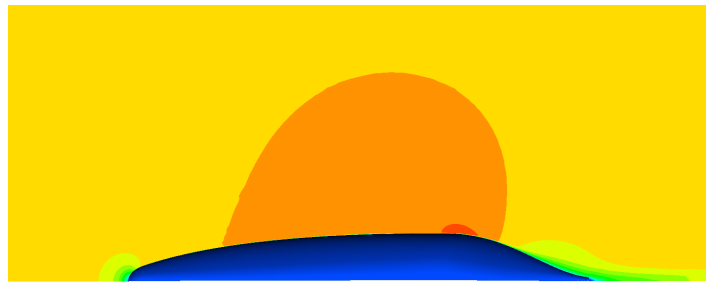

(b) Design 2 - Natural Laminar Flow (NLF) body

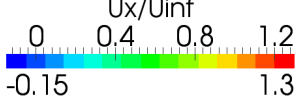

Fig. 8. Stream-wise velocity contours for two different AUV hull shapes. Flow from left to right, local curvature causing flow acceleration implies change of the pressure gradient likely to encourage transition to turbulence.

boundary layer and a wide wake. Both of these effects lead to approximately $50 \%$ increase in drag compared to design 2 .

It is also vital to understand how important the natural transition is in governing the performance of the hull. In a real environment the vehicle will be subject to various free-stream turbulence levels, surface roughness and assembly imperfections, all of which may trip the flow to become turbulent earlier than the idealised CFD result would suggest. Table II presents the force coefficients for designs 0 and 2 computed using the $k-\omega S S T$ model, which leads to the transition occurring almost immediately. This causes the Myring design to produce $15 \%$ less drag when subject to an angle of incidence of $3^{\circ}$ than the NLF body. This is mainly due to the lower wetted surface area for the same useful enclosed volume of the former concept. Overall, both designs may be seen to exhibit much higher drag in the fully turbulent scenario.
TABLE II

FORCE COEFFICIENTS FOR THE CHOSEN PRELIMINARY HULL SHAPE DESIGNS ASSUMING FULLY TURBULENT FLOW (DEFINED USING $(\Delta / \rho)^{\frac{2}{3}}$ ) AT AN ANGLE OF ATTACK OF $3^{\circ}$.

\begin{tabular}{|c|c|c|c|c|}
\hline Design & $\mathrm{C}_{\mathrm{D}}$ & $\mathrm{C}_{\mathrm{Di}}$ & $\mathrm{C}_{\mathrm{L}}$ & $\mathrm{L} / \mathrm{D}$ \\
\hline \multicolumn{5}{|c|}{$\operatorname{Re} 1.5$ million } \\
\hline 0 & $8.31 \mathrm{E}-3$ & $2.58 \mathrm{E}-4$ & $-2.06 \mathrm{E}-4$ & -0.02 \\
\hline 2 & $7.20 \mathrm{E}-3$ & $1.03 \mathrm{E}-4$ & $1.79 \mathrm{E}-3$ & 0.24 \\
\hline \multicolumn{5}{|c|}{ Re 3.0 million } \\
\hline 0 & $7.26 \mathrm{E}-3$ & $3.22 \mathrm{E}-4$ & $-7.75 \mathrm{E}-4$ & -0.10 \\
\hline 2 & $6.29 \mathrm{E}-3$ & $6.39 \mathrm{E}-3$ & $1.70 \mathrm{E}-3$ & 0.27 \\
\hline
\end{tabular}

\section{B. Appended hull characteristics}

Based on the preliminary design concept exploration and a parallel study investigating the design of the hydrofoils [29], three candidate shapes for the final design were considered. These were based on the NLF hull concept (design 2), given it has been found to offer favourable characteristics in the more realistic natural transition scenario and performed nearly as well as its competitors in the pessimistic fully turbulent case. A vertical stabiliser fin was also added and additional fairings accommodating sensor payloads and structural elements were also integrated into the upper part of the hull. A segment of parallel mid-body also had to be accommodated in order to allow easier integration of the cylindrical pressure vessel. This resulted in designs 4,5 , and 6 being created. The major differences between them were the shape of the after body affecting the wake and laminar separation. Lift and drag characteristics for each design were then computed using CFD across a range of expected speeds and at the target angle of attack of $3^{\circ}$. Length-based Reynolds number corresponding to the target maximum speed is approximately 1.5 million and 750,000 for the nominal velocity the glider is envisaged to adopt during most of its service.

Figure 9 presents the predicted lift-to-drag ratios for all of the developed designs. The scenario where transition is allowed to occur naturally allowed each of the vehicles to achieve much lower drag than in the fully turbulent evaluation, leading to significantly higher L/D. Furthermore, due to its stern shape allowing a more gentle pressure recovery design 0 has outperformed its competitors noticeably. In the fully turbulent case the differences between all of the shapes were minimal as their wetted surface areas were very comparable. It should be noted that most of the lift was generated by the hydrofoils and hence most of the observed differences are due to a difference in drag induced by the hulls. The presented $\mathrm{L} / \mathrm{D}$ estimates also indicate that even in the most pessimistic scenario the new glider should be able to at least match its competitors in terms of achieving the preferred glide path range between 30 and $40^{\circ}$.

Based on the results presented a candidate shape of the glider has been proposed, as shown in Figure 10. It is virtually identical to the best-performing design 0 except the longitudinal position of hydrofoils has been adjusted from the baseline configuration to provide more favourable pitch balance. 


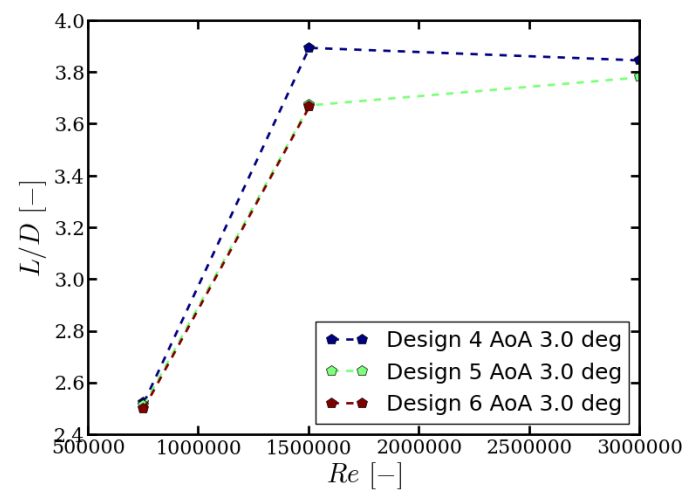

(a) Free transition

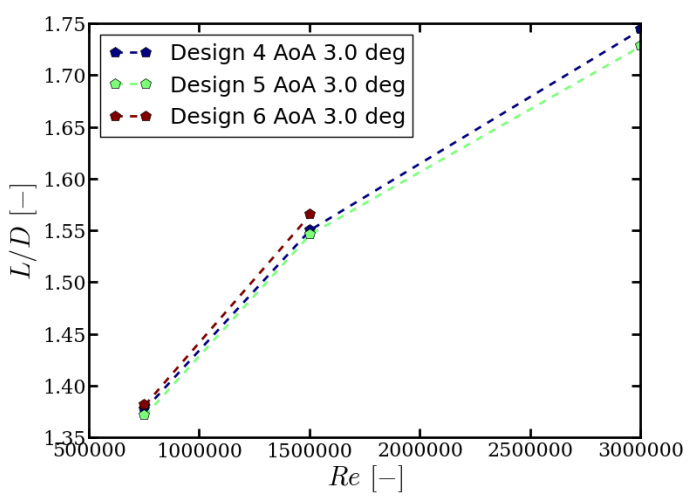

(b) Turbulent

Fig. 9. Lift to drag ratios predicted for the three intermediate designs.

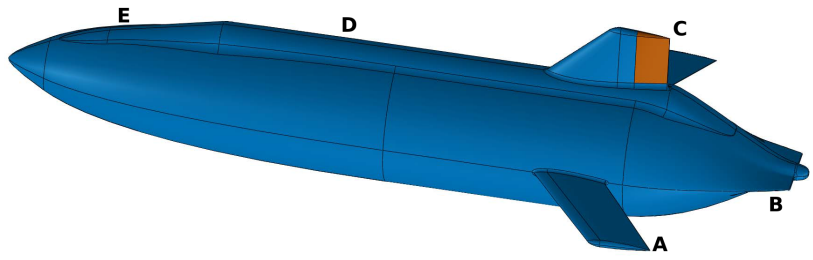

Fig. 10. Final underwater glider design evaluated; A - hydrofoil, B - horizontal stabiliser, C - tail fin (movable part highlighted in orange), D - central fairing for structural frame, E - fairing for a CTD sensor.

\section{Selected design performance prediction}

Figure 11 shows the change of non-dimensional drag on the glider as a function of Reynolds number and angle of attack. It is apparent that if the flow is assumed to be fully turbulent the resistance increases, primarily due to the nearly thee-fold increase of the friction drag. At the same time, assuming natural transition leads to an increase of pressure drag by $8 \%$ compared to the fully turbulent scenario, but this penalty is small compared to the viscous drag coefficient change.

Rudnick et al. [1] reported that the current Slocum and Spray gliders are characterised by approximately constant drag area over a range of operational Reynolds numbers, while the Seaglider exhibits a reduction in the drag coefficient as the Reynolds number increases which is caused by its natural

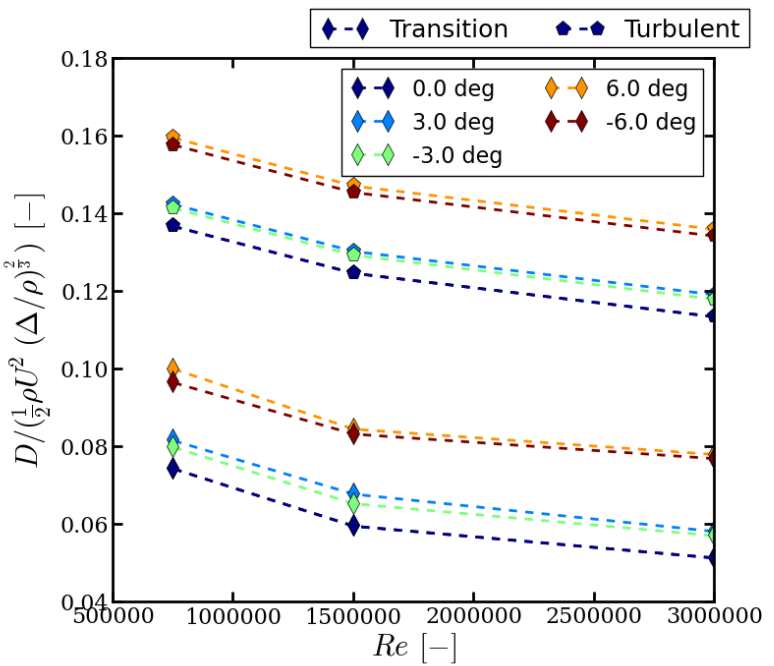

Fig. 11. Drag area estimate for the final design as a function of Reynolds number assuming both natural transition and fully turbulent flow. The influence of induced drag at the design angle of attack of $3^{\circ}$ and higher $6^{\circ}$ also indicated.

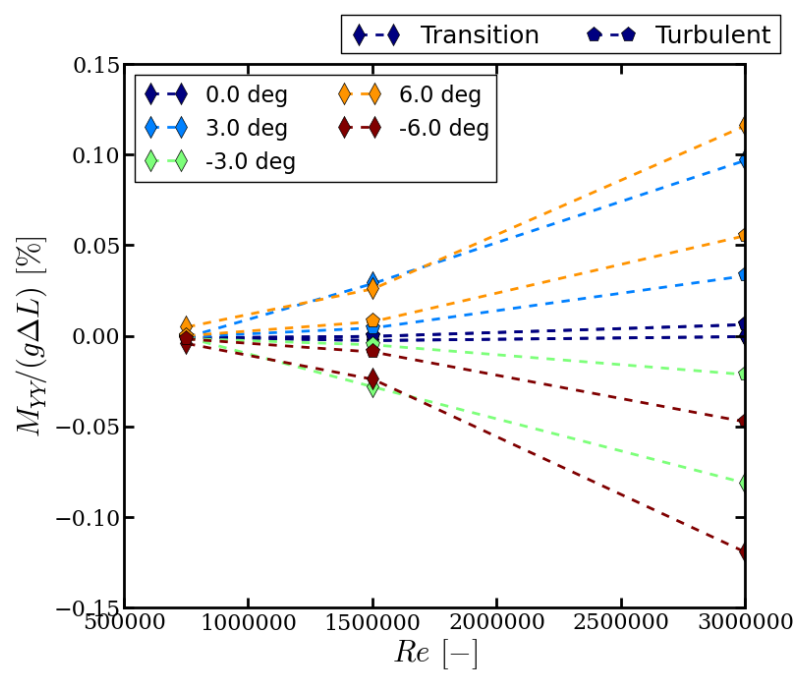

Fig. 12. Pitch moment coefficient about the centre of gravity acting on the final design shown as a function of Reynolds number and angle of attack.

laminar flow hull. In the present datasets a slight reduction in drag coefficient is seen as a function of Reynolds number, although the predicted curve has a lower slope than was reported for the Seaglider and appears to reach a plateau as the vehicle nears its maximum speed. It is also worth noting that the induced drag coefficient varies slightly for the present hull depending on whether positive or negative angles of attack are adopted. This is caused by the top-bottom asymmetry of the AUV caused by the presence of the central sensor fairing and the vertical fin on the upper side of the hull.

In the selected design, the pitch stability has been regarded as a crucial hydrodynamic characteristic and substantial care was put into placing the hydrofoils at a favourable longitudinal 
position. The target result was to achieve neutral pitch balance at the nominal velocity so that the pitch actuation control can rely primarily on adjusting the hydrostatic balance. At higher speeds the glider system delivers a restoring moment which should help in pointing it into the flow and thus reducing the actuation required of the movable mass system. This data is shown in Figure 12. Non-dimensionalisation chosen to reflect the magnitude of the moment generated at each speed relative to the size of the movable mass system.

From the operational perspective, the performance of an underwater glider may be expressed as its ability to achieve the highest forward speed for a fixed amount of buoyancy change. In order to increase the endurance of the vehicle it is also desirable to reduce the glide path angles so that the ratio of forward to vertical velocity achieved is as large as possible.

In order to compute these performance characteristics one may assume a range of pitch angles that a glider could adopt thanks to its ability to shift the centre of gravity position laterally and find a point at which the forces acting on the system are in equilibrium. This reduces the problem to one only having two free variables, horizontal and vertical speed, the ratio of which affects the real glide path angle which is different to the assumed pitch inclination (see Figure 1).

Figure 13 presents this data for the proposed final glider shape subject to three different vertical forces corresponding to minimum, nominal, and maximum intended actuation of the buoyancy engine. The lift and drag coefficients were computed using CFD as a function of the angle of attack and Reynolds number using the transition and fully turbulent RANS models. The simplified equations of motion were then solved numerically using the regressed CFD data until balance of forces for each pitch angle has been found.

Notably, that the predictions made using the transition model yield a much more optimistic estimate of forward speeds of the glider, mainly due to the reduced drag. Moreover, the data suggests shallower glide path angles may be obtained in the natural transition scenario with near-optimum forward velocities being reached at angles about of $30^{\circ}$, compared to $40^{\circ}$ for the fully turbulent results.

It has also been confirmed that the design should be able to achieve the desired maximum speed with buoyancy engine capacity within the range used by the presently used underwater gliders (these have reported ballast fractions of Slocum - 0.87\%, Spray - $1.76 \%$ and Seaglider - $1.62 \%$ of total vehicle mass [1]). The present estimates do not include the added drag due to sensors which, as already pointed out, may be significant. Present estimates indicate, however, that sufficient margin exists in the design to still allow the speed requirements to be met even if the drag increases substantially.

It is worth comparing pressure and skin-friction coefficient distributions for the final design computed using both the transition model and the "fully turbulent" approach in Figures 15 and 14. These were calculated for the maximum target glide speed and the nominal angle of attack of $3^{\circ}$. One can note that the pressure distributions are very comparable in both cases, with only noticeable differences occurring around the stern in the pressure recovery region. The skin friction coefficient has been predicted to have an overall similar distribution but the SST model predicted higher viscous forces over most of the hull, yielding a higher integral value. This further corroborates the observations made based on total force coefficients.

Figure 16 presents iso-contours of turbulence intensity around the hull at the maximum glide speed and nominal angle of attack coloured by eddy viscosity. The latter highlights the regions in which the RANS model is particularly active and affects the overall flow solution. If free transition is considered, high turbulence intensity is not encountered until the fluid reaches the parallel mid-body and transitions due to the lack of a favourable pressure gradient. When the SST model is used, however, turbulent kinetic energy (TKE) production in the boundary layer contributes to high TKE levels from relatively early on in terms of local Reynolds number.

Figure 17 depicts streamlines computed for fluid particles close to the centreline of the AUV at the maximum glide speed and nominal angle of attack of $3^{\circ}$. These are coloured by the local pressure coefficient. One can note how the flow at the underside of the AUV sees continuous favourable pressure gradient up to the stern which is expected to have a beneficial effect on encouraging natural laminar flow. On the upper side of the vehicle adverse pressure gradient starts to occur approximately at amidships. An important observation is the inception of a vortex at the radius between the central fairing and the hull surface which starts to take place around the leading edge of the vertical fin. It is believed that noticeable drag reduction will be possible through careful detailed design of this part of the AUV in the future.

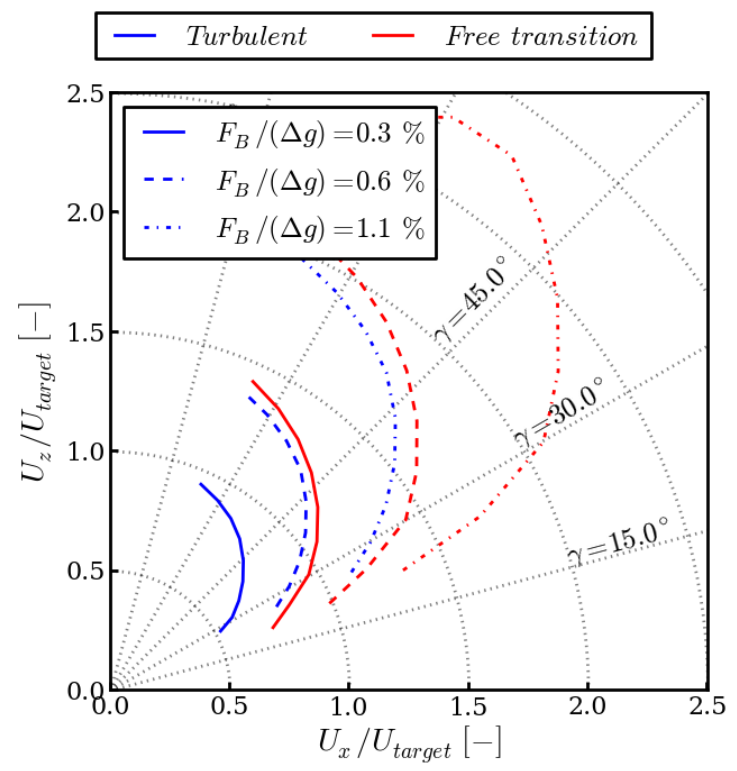

Fig. 13. Performance envelopes for the proposed underwater glider design as computed using data from two different turbulence models. $F_{B}$ denotes a constant vertical force induced by a buoyancy change. 


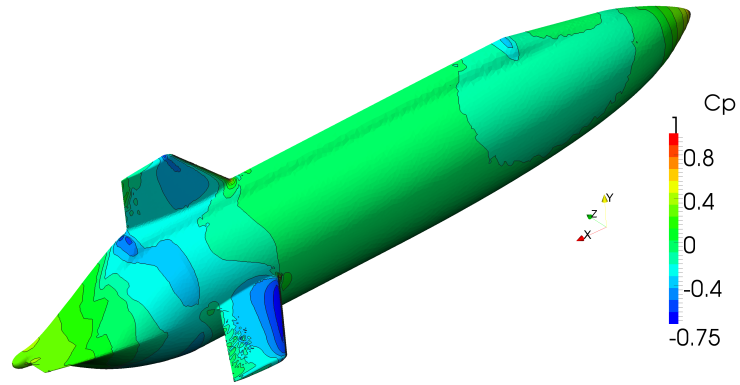

(a) Natural transition $-k_{L}-k_{T}-\omega$

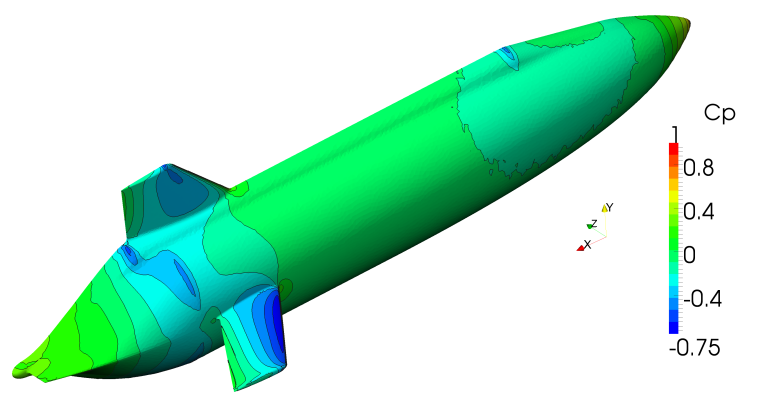

(b) $k-\omega S S T$

Fig. 14. Pressure coefficient distribution on the final design at an angle of attack of $3^{\circ}$ and $R e_{L} 1.5$ million predicted using two turbulence models.

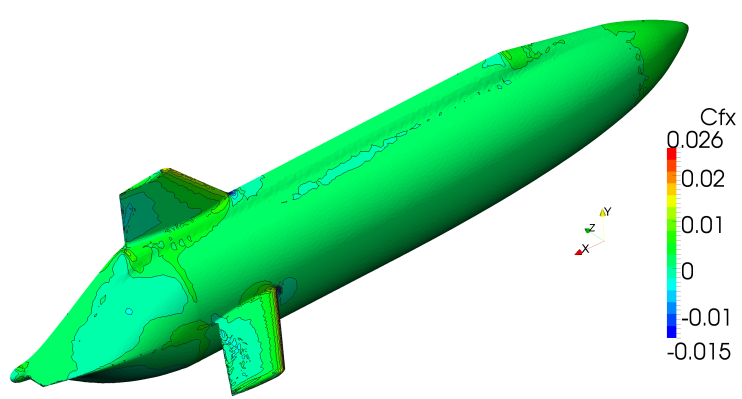

(a) Natural transition $-k_{L}-k_{T}-\omega$

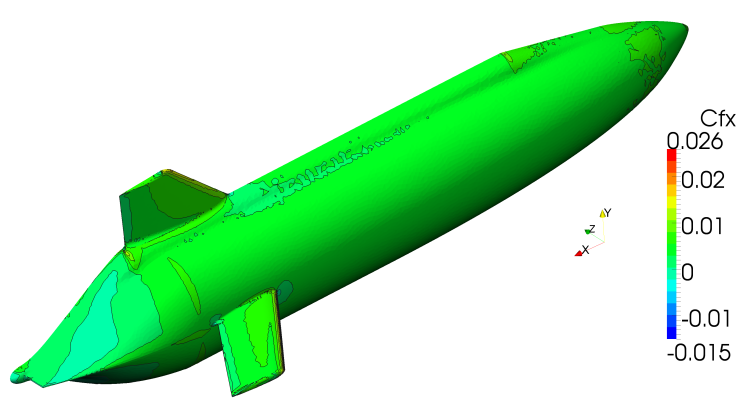

(b) $k-\omega S S T$

Fig. 15. Skin-friction coefficient distribution on the final design at an angle of attack of $3^{\circ}$ and $R e_{L} 1.5$ million predicted using two turbulence models.

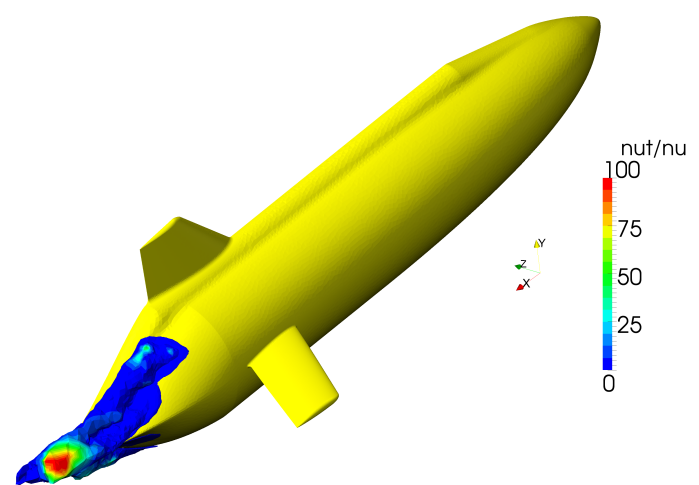

(a) Natural transition $-k_{L}-k_{T}-\omega$

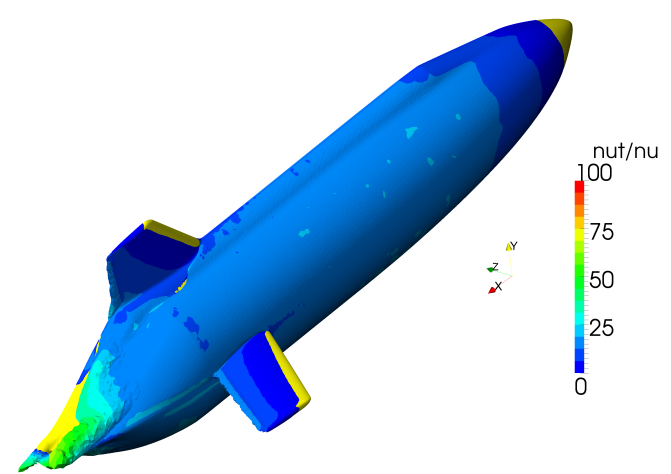

(b) $k-\omega S S T$

Fig. 16. Iso-contour of turbulence intensity of $5 \%$ coloured by nondimensional eddy viscosity predicted for the final design at an angle of attack of $3^{\circ}$ and $\operatorname{Re}_{L} 1.5$ million using two turbulence models.

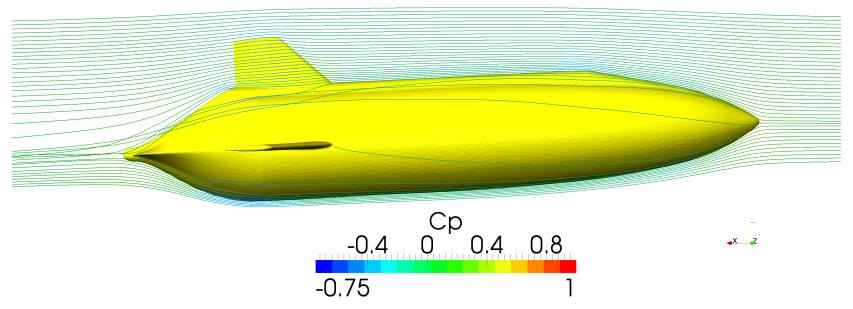

Fig. 17. Streamlines computed for the final design at an angle of attack of $3^{\circ}$ and $R e_{L} 1.5$ million using the transition model.

\section{CONCLUSIONS}

It has been shown that computational fluid dynamics offer a useful tool in performing preliminary hydrodynamic analysis of underwater glider designs. Despite a relatively high cost per simulation, availability of high-power computing resources allowed the presented calculations to aid in developing a concept design for a new vehicle. The predictions also highlighted key areas that will require attention at future design stages.

The findings highlight the potential performance gains possible to achieve by encouraging natural laminar flow and by drag reduction in general. While it is possible to compensate for a drag increase with the use of larger, more powerful hydrofoils, only a fraction of their lift gets translated into useful forward thrust, particularly if shallower glide path angles are adopted. Majority of the lift is instead used to resist 
the upward motion and therefore such a solution requires the use of heavier and less energy efficient buoyancy engines.

In practice, however, achieving natural laminar flow may prove difficult. Firstly, because it requires the overall shape of the design to be dictated by hydrodynamics, which often clashes with the cylindrical profile of a typical pressure vessel, for instance. Furthermore, an underwater glider is first and foremost a sensor platform. However, many scientific payloads, such as conductivity, temperature and depth (CTD) sensors or turbulence probes need to be pointed into the flow through protrusions in the vehicle hull. These act locally to generate turbulence and make maintaining laminar flow challenging. Finally, in order to maintain favourable hydrodynamic characteristics in service careful rules for assembly, disassembly, repair and maintenance would be required of the operators to ensure local imperfections stimulating early transition do not accumulate over time.

These observations show that while encouraging natural laminar flow on underwater gliders may appear like an obvious design direction, it is faced with serious obstacles. It is therefore important to develop further understanding of how the practical design aspects may be dealt with in order to increase performance of next generation of this class of marine vehicles. Nonetheless, the currently proposed glider shape appears to offer satisfactory performance while making use of the natural laminar flow and still meeting constraints imposed by the mechanical design of the internal parts of the vehicle. It is thus hoped that as the design becomes refined and ultimately built it will meet its operational requirements.

\section{ACKNOWLEDGMENTS}

The presented work has been funded as a part of the BRIDGES Project (http://www.bridges-h2020.eu/). This Project has received funding from the European Unions Horizon 2020 research and innovation programme under grant agreement No 635359. The authors wish to acknowledge the use of the IRIDIS High Performance Computing Facility, and associated support services at the University of Southampton, in the completion of this work and the use of OpenFOAM (http://openfoam.org/) for all of the presented simulations.

\section{REFERENCES}

[1] D. L. Rudnick, R. E. Davis, C. C. Eriksen, D. M. Fratantoni, and M. J. Perry, "Underwater Gliders for Ocean Research," Marine Technology Society Journal, vol. 38, no. 2, pp. 73-84, 2004.

[2] S. Wood, Autonomous Underwater Gliders. Vienna, Austria: INTECH Open Access Publisher, 2009.

[3] G. Griffiths, C. P. Jones, J. Ferguson, and N. Bose, "Undersea gliders," Journal of Ocean Technology, vol. 2, no. 2, pp. 64-75, 2007.

[4] J. G. Graver, Underwater gliders - dynamics, control and design. $\mathrm{PhD}$ thesis, Princeton University, 2005.

[5] C. C. Eriksen, "Autonomous Underwater Gliders," in Autonomous and Lagrangian Platforms and Sensors (ALPS) Workshop, (31 March - 2 April, Sea Lodge, La Jolla, CA, USA), pp. 1-5, 2003.

[6] J. Sherman, R. E. Davis, W. B. Owens, and J. Valdes, "The autonomous underwater glider," Oceanic Engineering, IEEE Journal of, vol. 26, no. 4, pp. 437-446, 2001.

[7] R. E. Davis, C. C. Eriksen, and C. P. Jones, "Autonomous Buoyancydriven Underwater Gliders," The technology and applications of autonomous underwater vehicles, pp. 37-58, 2002.
[8] M. Rosenfeld, M. Wolfshtein, and M. Israeli, "A numerical study of the laminar incompressible flow over a 6:1 prolate spheroid at 10 degrees incidence," Int. J. for Numerical Methods in Fluids, vol. 15, no. December, pp. 147-173, 1992.

[9] C. C. Eriksen, T. J. Osse, R. D. Light, T. Wen, T. W. Lehman, P. L. Sabin, J. W. Ballard, and A. M. Chiodi, "Seaglider : A Long-Range Autonomous Underwater Vehicle for Oceanographic Research," IEEE Journal of Oceanic Engineering, vol. 26, no. 4, pp. 424-436, 2001.

[10] A. B. Phillips, S. R. Turnock, and M. Furlong, "The use of computational fluid dynamics to aid cost-effective hydrodynamic design of autonomous underwater vehicles," Proc. IMechE Part M: J. Engineering for the Maritime Environment, vol. 224, pp. 239-254, 2010.

[11] R. M. Hubbard, "Hydrodynamics technology for an advanced expendable mobile target (AEMT)," tech. rep., Applied Physics Laboratory, University of Washington, Washington, DC, USA, 1980.

[12] A. Alvarez, A. Caffaz, A. Caiti, G. Casalino, L. Gualdesi, A. Turetta, and R. Viviani, "Folaga: A low-cost autonomous underwater vehicle combining glider Fo and AUV capabilities," Ocean Engineering, vol. 36, pp. 24-38, 2009.

[13] P. Jagadeesh, K. Murali, and V. G. Idichandy, "Experimental investigation of hydrodynamic force coefficients over AUV hull form," Ocean Engineering, vol. 36, pp. 113-118, 2009.

[14] S. A. Malik and P. Guang, "Transient Numerical Simulations for Hydrodynamic Derivatives Predictions of an Axisymmetric Submersible Vehicle," Research Journal of Applied Sciences, Engineering and Technology, vol. 5, no. 21, pp. 5003-5011, 2013.

[15] A. Phillips, M. Furlong, and S. R. Turnock, "The Use of Computational Fluid Dynamics to Assess the Hull Resistance of Concept Autonomous Underwater Vehicles," in Oceans 2007-Europe, 2007.

[16] J. V. N. de Sousa, A. R. L. de Macedo, W. F. de Amorim Junior, and d. L. A. G. B, "Numerical Analysis of Turbulent Fluid Flow and Drag Coefficient for Optimizing the AUV Hull Design," Open Journal of Fluid Dynamics, vol. 4, no. September, pp. 263-277, 2014.

[17] S. Tang, T. Ura, T. Nakatani, B. Thornton, and T. Jiang, "Estimation of the hydrodynamic coefficients of the complex-shaped autonomous underwater vehicle TUNA-SAND," Journal of Marine Science and Technology, vol. 14, no. 3, pp. 373-386, 2009.

[18] J. S. Parsons, R. E. Goodsont, and F. R. Goldschmiedt, "Shaping of Axisymmetric Bodies for Minimum Drag in Incompressible Flow," Journal of Hydronautics, vol. 8, no. 3, pp. 100-107, 1974.

[19] R. B. Langtry, A correlation-based transition model using local variables for unstructured parallelized CFD codes. $\mathrm{PhD}$ thesis, University of Stuttgart, 2006.

[20] J. Dantas and E. de Barros, "Numerical analysis of control surface effects on AUV manoeuvrability," Applied Ocean Research, vol. 42, pp. 168 $181,2013$.

[21] D. K. Walters and D. Cokljat, "A Three-Equation Eddy-Viscosity Model for Reynolds-Averaged NavierStokes Simulations of Transitional Flow," Journal of Fluids Engineering, vol. 130, no. 12, p. 121401, 2008.

[22] Coupland, P. E. Roach, D. H. Brierley, and I. Ryhming, "ERCOFTAC T3 Case," 1990.

[23] M. V. Ol, B. R. McCauliffe, E. S. Hanff, U. Scholz, and C. Kähler, "Comparison of Laminar Separation Bubble Measurements on a Low Reynolds Number Airfoil in Three Facilities," 35th AIAA Fluid Dynamics Conference and Exhibit, pp. 1-11, 2005.

[24] S. S. Dodbele and C. P. van Dam, "Design of fuselage shapes for Natural Laminar Flow," tech. rep., Vigyan Research Associated Inc., NASA Contractor Report 3970, Hampton, VA, 1986.

[25] F. Menter, "Two-equation eddy-viscosity turbulence models for engineering applications," AIAA Journal, vol. 32, no. 8, pp. 1598-1605, 1994.

[26] I. Pointwise, "Pointwise," 2016.

[27] M. S. Selig, J. J. Guglielmo, A. P. Broeren, and P. Giguere, "Summary of Low-speed airfoil data, volume 1," tech. rep., SoarTech Publications, Virginia Beach, Virginia, 1995.

[28] D. F. Myring, "A theoretical study of the effects of body shape and Mach number on the drag of bodies of revolution in subcritical axisymmetric flow," tech. rep., Royal Aircraft Establishment, Farnborough, Hants, 1981.

[29] S. Lemaire, A. K. Lidtke, G. Vaz, and S. R. Turnock, "Modelling Natural Transition on Hydrofoils for Application in Underwater Gliders," in 19th Numerical Towing Tank Symposium (NuTTS), (3-4 October, St Pierre d'Oleron, France), 2016. 